\title{
Revista de Medicina Veterinaria
}

January 2017

\section{Aproximación físico-química a las alteraciones ácido base en la orina de ovinos con alcalosis hipoclorémica}

\author{
David Alexander Martínez Rodríguez \\ Universidad Nacional de Colombia, damartinezr@unal.edu.co \\ Olimpo Juan Oliver Espinosa \\ Universidad Nacional de Colombia, ojolivere@unal.edu.co
}

Follow this and additional works at: https://ciencia.lasalle.edu.co/mv

\begin{abstract}
Citación recomendada
Martínez Rodríguez DA y Oliver Espinosa OJ. Aproximación físico-química a las alteraciones ácido base en la orina de ovinos con alcalosis hipoclorémica. Rev Med Vet. 2017;(35): 113-127. doi: https://doi.org/ $10.19052 / \mathrm{mv} .4394$
\end{abstract}

This Artículo de Investigación is brought to you for free and open access by the Revistas científicas at Ciencia Unisalle. It has been accepted for inclusion in Revista de Medicina Veterinaria by an authorized editor of Ciencia Unisalle. For more information, please contact ciencia@lasalle.edu.co. 


\title{
Aproximación físico-química a las alteraciones ácido base en la orina de ovinos con alcalosis hipoclorémica
}

\author{
David Alexander Martínez Rodríguez ${ }^{1}$ / Olimpo Juan Oliver Espinosa ${ }^{2}$
}

1 Médico veterinario. Departamento de Fisiología, Facultad de Medicina, Universidad Nacional de Colombia, sede Bogotá.

$凶$ damartinezr@unal.edu.co

2 Médico veterinario. MSc. DVSc, Clínica para Grandes Animales, Departamento de Salud Animal, Facultad de Medicina Veterinaria y Zootecnia, Universidad Nacional de Colombia, sede Bogotá. Director del grupo de investigación en Enfermedades de Grandes Animales Medicina.

$\bowtie$ ojolivere@unal.edu.co

Cómo citar este artículo: Martínez Rodríguez DA, Oliver Espinosa OJ. Aproximación físico-química a las alteraciones ácido base en la orina de ovinos con alcalosis hipoclorémica. Rev Med Vet. 2017;(35):113-27. doi: http://dx.doi.org/10.19052/mv.4394

\begin{abstract}
Resumen
La aproximación físico-química para determinar el pH urinario es relativamente nueva y no se ha usado en condiciones patológicas en animales. En el presente artículo, el objetivo principal fue demostrar la validez de esta teoría en la orina de ovinos con alcalosis metabólica hipoclorémica. Se realizó un estudio de tipo experimental para la inducción de la alcalosis metabólica hipoclorémica en ovinos. Durante el periodo de control y de inducción se determinaron en la orina: $\mathrm{pH}$ calculado, $\mathrm{pH}$ medido, excreción neta de ácido, amonio y diferencia de iones fuertes en la orina (SID) cada $24 \mathrm{~h}$ hasta el desarrollo de la aciduria paradójica o el deterioro físico de los sujetos. Se determinó la correlación de Pearson $(\rho)$ entre el $\mathrm{pH}$ medido y calculado a partir del modelo del SID en la orina. Se observó una correlación alta entre el $\mathrm{pH}$ urinario medido y el calculado usando el SID calculado con base en la excreción neta de ácido $(\rho=0,874)$. La correlación entre SID calculado y $\mathrm{pH}$ en orina fue significativa $(\rho=0,839)$. Sin embargo, la correlación entre el SID y el $\mathrm{pH}$ medido de la orina fue moderada $(\rho=0,588)$. Se concluye que existe una alta correlación entre el pH calculado a partir del SID usando la excreción neta de ácido y el $\mathrm{pH}$ medido en la orina de ovinos con alcalosis metabólica hipoclorémica. Esto indica que el $\mathrm{pH}$ urinario depende fuertemente del SID y, por lo tanto, la reducción en el $\mathrm{pH}$ puede ser explicada por la disminución del SID.
\end{abstract}

Palabras clave: alcalosis metabólica, aciduria paradójica, aproximación físico-química, diferencia de iones fuertes, excreción neta de ácido, ovinos.

\section{Physical-chemical approach to acid-base alterations in the urine of sheep with hypochloremic alkalosis}

\begin{abstract}
The physical-chemical approach to determine urinary $\mathrm{pH}$ is relatively new and has not yet been used in pathological conditions in animals. The main objective of this paper was to demonstrate the validity of this theory in the urine of sheep with hypochloremic metabolic alkalosis. An experimental-type study was conducted to induce hypochloremic metabolic alkalosis in sheep. During the control and induction periods, calculated $\mathrm{pH}$, measured $\mathrm{pH}$, net acid excretion, ammonium and strong ion difference (SID) in urine were examined every 24 hours until development of paradoxical aciduria or physical deterioration of subjects. Pearson's correlation $(\rho)$ was determined between measured and calculated $\mathrm{pH}$ based on SID in urine. A high correlation between measured and calculated urine $\mathrm{pH}$ was observed using SID calculated from net acid excretion $(\rho=0.874)$. The correlation between calculated SID and urine $\mathrm{pH}$ was significant $(\rho=0.839)$. However, the correlation between SID and measured urine $\mathrm{pH}$
\end{abstract}


was moderate $(\rho=0.588)$. It is concluded that there is a high correlation between $\mathrm{pH}$ calculated from SID using net acid excretion and $\mathrm{pH}$ measured in the urine of sheep with hypochloremic metabolic alkalosis. This indicates that urine $\mathrm{pH}$ depends strongly on SID and, therefore, a reduction in $\mathrm{pH}$ can be explained by a decrease in SID.

Keywords: metabolic alkalosis, paradoxical aciduria, physico-chemical approach, strong ion difference, net acid excretion, sheep.

\title{
Aproximação físico-química às alterações ácido base na urina de ovinos com alcalose hipoclórica
}

\begin{abstract}
Resumo
A aproximação físico-química para determinar o pH urinário é relativamente nova e não tem sido usado em condições patológicas em animais. Neste artigo, o objetivo principal foi demonstrar a validez desta teoria na urina de ovinos com alcalose metabólica hipoclórica. Realizou-se um estudo de tipo experimental para a indução da alcalose metabólica hipoclórica em ovinos. Durante o período de controle e de indução determinaram na urina: $\mathrm{pH}$ calculado, $\mathrm{pH}$ medido, excreção neta de ácido, amônio e diferença de íons fortes na urina (SID) cada 24 horas hasta o desenvolvimento da acidúria paradoxal ou a deterioração física dos sujeitos. Determinou-se a correlação de Pearson ( $\rho$ ) entre o pH medido e calculado a partir do modelo do SID na urina. Observou-se uma correlação alta entre o $\mathrm{pH}$ urinário medido e o calculado usando o SID calculado a partir da excreção líquida de ácido $(\rho=0,874)$. A correlação entre SID calculado e pH em urina foi significativa $(\rho=0,839)$. Contudo, a correlação entre o SID e o $\mathrm{pH}$ medido da urina foi moderada $(\rho=0.588)$. Conclui-se que existe uma alta correlação entre o $\mathrm{pH}$ calculado a partir do SID usando a excreção líquida de ácido e o $\mathrm{pH}$ medido na urina de ovinos com alcalose metabólica hipoclórica. Isto indica que o $\mathrm{pH}$ urinário depende fortemente do SID e, portanto, a redução no $\mathrm{pH}$ pode ser explicada pela diminuição do SID.
\end{abstract}

Palavras chave: alcalose metabólica, acidúria paradoxal, aproximação físico-química, diferencia de íons fortes, excreção líquida de ácido, ovinos.

\section{INTRODUCCIÓN}

En el paradigma interpretativo del modelo de Henderson-Hasselbalch (H-H), la alcalosis metabólica en humanos y animales se caracteriza por un aumento en el $\mathrm{pH}$ plasmático, con aumento en las concentraciones de bicarbonato. Este modelo ha identificado dos fases de la alcalosis metabolica: la de generación y la de mantenimiento (1-5). La generación requiere que ocurra la pérdida de $\mathrm{H}^{+}$o la ganancia de $\mathrm{HCO}_{3}(6,7)$. En rumiantes la pérdida de ácido ocurre comúnmente en desplazamien- to o vólvulo abomasal, indigestión vagal u obstrucciones intestinales proximales; mientras la ganancia de $\mathrm{HCO}_{3}$ es poco común y se presenta cuando se adiciona bicarbonato o hidróxido de magnesio a las dietas como tratamiento $(8,9)$.

El mantenimiento de la alcalosis metabólica se da como consecuencia de la incapacidad renal para excretar el exceso de $\mathrm{HCO}_{3}$-filtrado (4,5,10-13). En rumiantes se ha identificado que los mecanismos que mantienen la alcalosis metabólica son la disminución en las concentracio- 
nes de $\mathrm{K}^{+}$y Cl plasmático y la reducción en el volumen circulante $(9,12,14-16)$. También se ha encontrado que la reducción en la concentración de $\mathrm{K}^{+}$que acompaña a la alcalosis metabólica puede ser una causa o consecuencia del mantenimiento de este desorden (4). La disminución en la concentración de $\mathrm{K}^{+}$puede generar alcalosis metabólica por la reducción en la tasa de filtración glomerular o la generación de acidosis intracelular $(4,9,17-19)$.

La excreción renal es un factor importante en el control del estado ácido base en pacientes con alcalosis metabólica, en la cual la regulación de la concentración de $\mathrm{H}^{+}$a través del mantenimiento de la concentración de los diferentes electrolitos es fundamental. Cuando un catión o un anión es excretado en mayor medida con respecto al otro, este es acompañado por la excreción de bicarbonato o de hidrogenión. Estos procesos fisiológicos dentro de los túbulos renales son los que determinan la excreción de $\mathrm{H}^{+}(5,9,20-23)$.

En relación con el paradigma físico-químico de la interpretación de los desórdenes ácido base, no hay hasta el momento estudios que muestren la aplicación de esta aproximación en la orina de pacientes con alcalosis metabólica hipoclorémica. Sin embargo, Constable y colaboradores, en 2009 (9), registraron la aplicación de la aproximación físico-química en la orina de bovinos y la relación de la diferencia de iones fuertes (SID) con el pH. Según esta aproximación, la ecuación general para la electroneutralidad en la orina está determinada de la siguiente manera (20):

$$
-(\mathrm{SID}-k)=\mathrm{AT}+\left[\mathrm{NH}_{4}\right]-\left[\mathrm{HCO}_{3}^{-}\right]
$$

Donde el SID corresponde a la diferencia entre la suma de los cationes y los aniones completamente disociados en la orina, bajo condiciones de $\mathrm{pH}$ entre 4,7 y 8,7 ; y $k$ corresponde al SID actual, cuando el $\mathrm{pH}$ es de 7,4 y AT a la acidez titulable de la orina (20).

Bajo un rango más estrecho de $\mathrm{pH}$, entre 5,0 y 8,0 , se puede derivar la ecuación que expresa la relación del $\mathrm{pH}$ con las siguientes variables independientes $\mathrm{SID},\left[\mathrm{NH}_{4}\right]$, $\left[\mathrm{PO}_{4}\right]$ y $\mathrm{P}_{\mathrm{CO} 2}$; y tres constantes: la constante de disociación del $\mathrm{H}_{2} \mathrm{CO}_{3}\left(\mathrm{~K}_{1}^{\prime}\right)$, la de disociación del $\mathrm{H}_{2} \mathrm{PO}_{4}\left(\mathrm{Ka}_{2}\right)$ y la solubilidad del $\mathrm{CO}_{2}$ en la orina $(S)$. De esta manera se obtiene la siguiente ecuación para el pH (20):

$$
\begin{gathered}
\mathrm{pH}=\log _{10}\left\{2\left([\mathrm{SID}]+\left[\mathrm{NH}_{4}\right]\right)\right\} /\left\{\left(\mathrm{K}_{1}^{\prime} \mathrm{SP}_{\mathrm{CO} 2}+\mathrm{Ka}_{2}\left[\mathrm{PO}_{4}\right]-\mathrm{Ka}_{2}\left([\mathrm{SID}]+\left[\mathrm{NH}_{4}\right]\right)+\left(\mathrm{K}_{1}^{\prime} \mathrm{S} \mathrm{P}_{\mathrm{CO} 2}+\mathrm{Ka}_{2}([\mathrm{SID}]+\right.\right.\right. \\
\left.\left.\left.\left.\left[\mathrm{NH}_{4}\right]+\mathrm{Ka}_{2}\left[\mathrm{PO}_{4}\right]\right) 2-4 \mathrm{Ka}_{2}{ }^{2}\left([\mathrm{SID}]+\left[\mathrm{NH}_{4}\right]\right)\left[\mathrm{PO}_{4}\right]\right\}\right\}^{1 / 2}\right\}
\end{gathered}
$$

Como en rumiantes, las concentraciones de fosfatos en la orina son menores a las de aniones fuertes, cationes fuertes y amonio. De hecho, son cercanas a 0 cuando se maneja un rango de $\mathrm{pH}$ entre 5,0 y 8,0. La ecuación anteriormente mencionada se puede simplificar de la siguiente manera (20):

$\mathrm{pH} \cong\left\{\mathrm{pK}_{1}^{\prime}-\log _{10}\left(\mathrm{SP}_{\mathrm{CO} 2}\right)\right\}+\log _{10}\left(\mathrm{SID}+\left[\mathrm{NH}_{4}\right]\right)$

Donde el SID corresponde a:
$\mathrm{SID}=\left[\mathrm{K}^{+}\right]+\left[\mathrm{Na}^{+}\right]+\left[\mathrm{Mg}^{+}\right]+\left[\mathrm{Ca}^{+}\right]-\left[\mathrm{Cl}^{-}\right]-\left[\mathrm{SO}_{4}^{2-}\right]$.

La ecuación descrita anteriormente solo será válida si la orina se mantiene dentro del rango de $\mathrm{pH}$ mencionado. Además de ello, esta ecuación indica que la concentración de bicarbonato urinario no afecta directamente el $\mathrm{pH}$, lo cual revela que esta es una variable dependiente en la orina al igual que en el plasma (20). La principal limitación de la aproximación físico-química en la orina es obtener valores exactos del SID, ya que la medi- 
ción de cada anión y catión por separado trae consigo un error propio en su medición (20). Teniendo en cuenta esta limitación, se estableció que la titulación de la orina proporciona un método acertado para la medición del SID, y así en el caso de bovinos puede ser calculado de la siguiente manera (20):

SID $=$ excreción neta de base $($ ENB $)+2,6=-$ excreción neta de ácido (ENA) + 2,6

Este modelo, desarrollado por Constable y colaboradores (9), predice que los valores de (SID $-k$ ) en la orina son equivalentes a la ENB o-ENA (20).

Con base en este modelo (20), el objetivo del presente estudio fue determinar la relación entre el $\mathrm{pH}$ urinario y el SID en ovinos con alcalosis metabólica hipoclorémica mediante el cálculo del SID a través de la ENA, en un modelo experimental de pérdida masiva de flujo abomasal por medio de una cánula duodenal.

\section{MATERIALES y MÉTODOS}

\section{Sujetos experimentales}

Un total de 8 ovinos, de raza criolla, hembras no preñadas, con edades entre 2 y 6 años, pesos entre 25 y $45 \mathrm{~kg}$, fueron usados en el experimento. Los animales fueron mantenidos en la Clínica de Grandes Animales de la
Universidad Nacional de Colombia, donde recibieron supervisión veterinaria continua. Fueron alimentados con pasto kikuyo (Pennisetum clandestinum) y agua a voluntad, junto con suplementación de $40 \mathrm{~g}$ por animal de sal mineralizada para ovinos (Ganasal Ovi-Caprinos ${ }^{\circledR}$ ).

Cada uno de los sujetos experimentales fue sometido a la implantación de una cánula duodenal en forma de $\mathrm{T}$ construida a base de nylon quirúrgico (figura 1), la cual consta de dos tapones, uno de los cuales permite el paso normal del flujo abomasal, y el otro genera bloqueo completo del flujo para la inducción de la alcalosis metabólica hipoclorémica. La cánula fue colocada por medio de laparotomía exploratoria por flanco derecho en el duodeno proximal, y adicionalmente se realizó la exteriorización de la arteria carótida en cada sujeto experimental $(2,19,23,24)$.

Los animales fueron mantenidos en el estudio bajo monitoreo médico veterinario las $24 \mathrm{~h}$, con el fin de identificar alteraciones patológicas durante la recuperación de la cirugía (14 días), más un periodo de adaptación (7 días), durante el proceso experimental, hasta que la condición de aciduria paradójica se reprodujera, o cuando las condiciones fisiológicas de cada uno de los sujetos se deterioraran (figura 2). En ese momento se decidió la realización de la eutanasia, la cual efectuó un médico veterinario con pentobarbital sódico vía endovenosa, previa tranquilización con xilazine.

Figura 1. Cánula duodenal en forma de T, en la que se observa el tapón que bloquea completamente el paso de contenido abomasal y permite el drenaje hacia el exterior del animal, para la generación de la alcalosis metabólica hipoclorémica

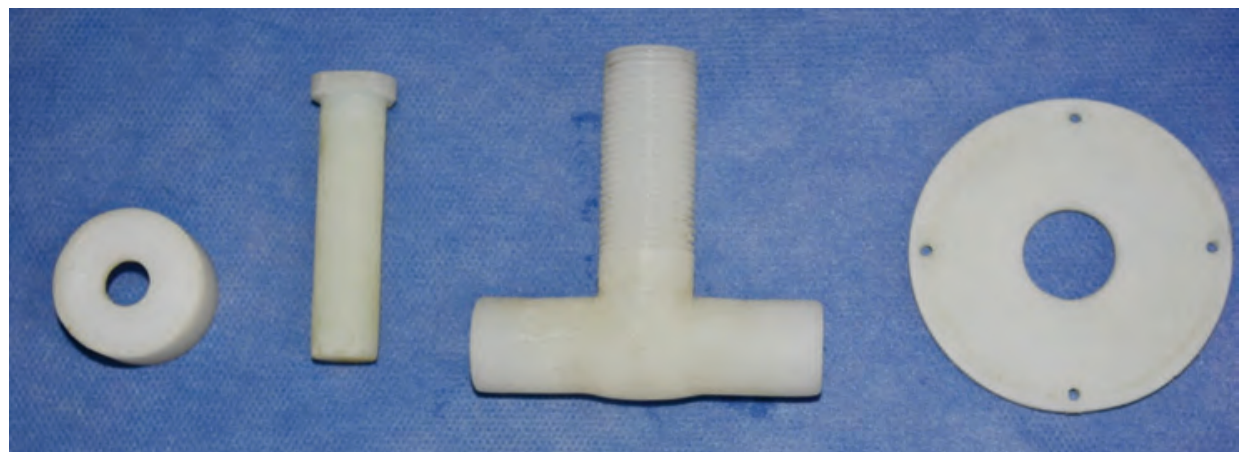


Figura 2. Esquema cronológico del diseño experimental que inicia con el procedimiento quirúrgico, seguido de la recuperación y adaptación, y finaliza con las fases experimentales de control e inducción de la alcalosis metabólica hipoclorémica

\begin{tabular}{|c|c|c|c|c|}
\hline $\begin{array}{l}\text { Procedimiento } \\
\text { quirúrgico }\end{array}$ & $\begin{array}{c}\text { Recuperación } \\
\text { quirúrgica }\end{array}$ & $\begin{array}{l}\text { Periodo de } \\
\text { adaptación }\end{array}$ & $\begin{array}{l}\text { Inicio del periodo } \\
\text { experimental } \\
\text { CONTROL }\end{array}$ & $\begin{array}{l}\text { Apertura de } \\
\text { la cánula } \\
\text { INDUCCIÓN }\end{array}$ \\
\hline
\end{tabular}

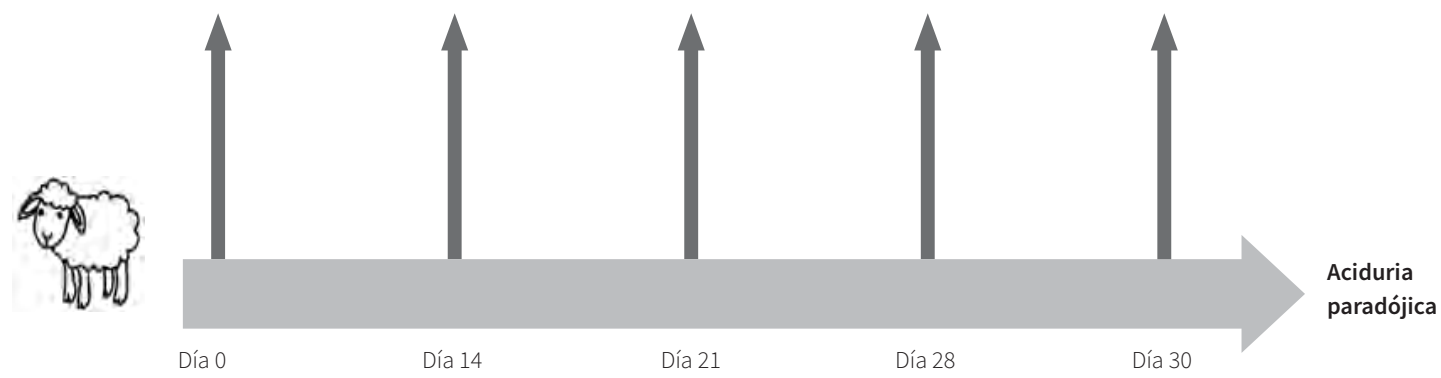

\section{Periodo experimental}

Se suspendió la administración de sal mineralizada una semana antes del inicio de la fase experimental. El periodo experimental se dividió en dos etapas (figura 2). La primera correspondió al control y se realizó por 48 h; durante este periodo se hizo la toma de muestras cada $24 \mathrm{~h}$ para las variables en la orina. La segunda correspondió a la apertura de la cánula y la inducción de la alcalosis metabólica hipoclorémica; durante esta etapa se tomaron muestras para las variables urinarias cada $24 \mathrm{~h}$. La toma de muestras fue suspendida cuando el sujeto experimental desarrolló aciduria, o cuando la condición física se deterioró.

\section{Toma de muestras}

Las muestras de orina fueron tomadas a las 2:00 p. m. todos los días cada día, momento durante el cual, según lo evidenciado por los resultados de ENA, son más confiables (9). Las muestras fueron tomadas por micción natural, y cuando esta no fue posible, se utilizó una sonda urinaria calibre 8 . Se recolectaron en beaker de plástico con tapa, y fueron procesadas durante las siguientes $4 \mathrm{~h}$. El restante se almacenó en tubos de criopreservación de $2 \mathrm{ml}$ y se congelaron a menos $-70{ }^{\circ} \mathrm{C}$ para su posterior medición (9).

\section{Procesamiento de muestras}

La determinación de la ENA ha sido registrada por diferentes autores $(3,4,21,25)$, pero para el presente estudio la acidez titulable y el amonio se calcularon con el método utilizado por Jörgensen y colaboradores (21), en el que se titula la orina manualmente. $\mathrm{El} \mathrm{pH}$ se midió por medio de un potenciómetro (Schott Lab $850^{\circledR}$, con electrodo Schott pH electrode Blue Line $\left.{ }^{\circledR}\right)$. El SID se calculó con base en la ENA $(\mathrm{SID}=\mathrm{ENB}+2,6=-\mathrm{ENA}+2,6)$ de acuerdo con lo evidenciado por Constable y colaboradores (9). Para el cálculo del $\mathrm{pH}$ urinario se usó la fórmula también utilizada por Constable y colaboradores (9), para $\mathrm{pH}$ urinario con rango entre 5 y $8(3,4,9,21,25)$.

\section{Análisis estadístico}

Para el presente estudio se realizó inicialmente un análisis de tipo descriptivo, usando promedios y medias para evaluar el comportamiento del $\mathrm{pH}$ durante la fase experimental, y luego análisis de correlación entre el $\mathrm{pH}$ medido y el $\mathrm{pH}$ calculado, basados en el SID calculado con la ENA, el SID calculado y el pH calculado y el SID calculado y el pH medido. Para esto se hizo una prueba de Friedman, con el objetivo de determinar si existieron o no diferencias significativas. Esta prueba se implementó por separado para los tiempos de control 
e inducción de la alcalosis metabólica con una p $\leq 0,05$. También se realizó la prueba de correlación de Pearson entre los siguientes pares de variables: el $\mathrm{pH}$ calculado y el medido, el SID calculado y el pH calculado y, finalmente, entre el SID calculado y el $\mathrm{pH}$ medido, usando una $\mathrm{p} \leq 0,01$.

El presente estudio experimental se llevó a cabo estrictamente según las normas estipuladas en la Ley 84 de 1989 y la Resolución 8430 de 1993 de la República de Colombia. Además, fue aprobado por el Comité de Ética de la Facultad de Medicina de la Universidad Nacional de Colombia, de acuerdo con el Acta de Evaluación 83 del 21 de junio de 2012.

\section{Resultados}

De los 8 sujetos experimentales, solo 7 fueron utilizados en el experimento, debido a que uno de ellos desarrolló peritonitis durante los 14 días de la fase de recuperación de la cirugía. Los registros obtenidos durante la fase tanto experimental de control como de inducción de la alcalosis metabólica fueron 56 lecturas, de la cuales 22 ocurrieron durante el periodo de control, y 34, durante el de inducción. Sin embargo, 3 de los sujetos experimentales presentaron dificultades en la apertura adecuada de la cánula al momento de la inducción, por lo que no se produjo pérdida de contenido abomasal y los datos de estas lecturas, que fueron 14 , se consideraron como parte del periodo de control, ya que no mostraron ningún tipo de alteración en ENB ni en AT ni en el equilibrio ácido base. Esto hizo que en tales sujetos experimentales el periodo de control durara 5 .

El periodo de inducción varió entre los sujetos experimentales. Se encontraron duraciones de $2 \mathrm{~d}$ para un sujeto; 4 para 2; 5 para $1 ; 6$ para 2, y 7 para 1 . De estos animales, solo 2 desarrollaron un $\mathrm{pH}$ ácido en la orina (aciduria paradójica) durante la fase de inducción. Se observó que aquellos animales en los cuales el tiempo de inducción tomó entre 6 y 7 días fueron los únicos que desarrollaron este fenómeno de aciduria paradójica.

Los sujetos experimentales mantuvieron un rango promedio de $\mathrm{pH}$ medido entre 8,313 (DE 0,089) y 8,403 (DE 0,040), mientras el rango promedio del $\mathrm{pH}$ calculado tuvo un rango entre $8,166(\mathrm{DE} 0,372)$ y $8,420(\mathrm{DE}$ $0,095)$ en el periodo de control. En la fase de inducción de la alcalosis metabólica previa al desarrollo del fenómeno de aciduria paradójica, el promedio de $\mathrm{pH}$ medido se mantuvo entre 8,200 (DE 0,123) y 8,459 (DE 0,076), mientras que el promedio calculado estuvo entre 8,317 $(D E 0,105)$ y 8,423 (DE 0,155). Finalmente, durante el desarrollo del fenómeno de aciduria, el rango promedio del pH medido bajó y se mantuvo entre 6,150 (DE 0) y 7,363 (DE 1,475), mientras el calculado fluctuó entre 7,041 (DE 0) y 7,645 (DE 1,145).

En la tabla 1 se ilustra el promedio de $\mathrm{pH}$ calculado y medido durante los días de control e inducción de la alcalosis metabólica durante el estudio, así como la diferencia que existe entre el $\mathrm{pH}$ calculado y el medido. Aquílas variaciones entre la diferencia absoluta mínima entre el $\mathrm{pH}$ calculado y el medido solo fueron de 0,25 , mientras que la diferencia máxima fue de 0,891 en las dos fases del estudio.

Adicionalmente, en la figura 3 se muestran las tendencias de los promedios tanto de $\mathrm{pH}$ calculado como medido a través del tiempo. Aquí se puede observar una clara orientación creciente en la diferencia absoluta por cada día que transcurre específicamente en el tiempo de inducción; es decir, a mayor tiempo durante la fase de inducción, el pH calculado se distancia del medido, como también se evidencia en la tabla 1 , con los valores absolutos, mientras que para los días de control las diferencias no muestran ninguna tendencia. 
Tabla 1. Promedios de $\mathrm{pH}$ urinario durante la fase experimental de control correspondiente a 5 días y la fase de inducción de la alcalosis metabólica hipoclorémica correspondiente a 7 días

\begin{tabular}{|c|c|c|c|c|c|}
\hline Fase & Promedio $\mathrm{pH}$ medido & $\sigma$ & Promedio $\mathrm{pH}$ calculado & $\sigma$ & Diferencia $\mathrm{pH}$ medido y $\mathrm{pH}$ calculado \\
\hline Día 1 C & 8,313 & 0,089 & 8,166 & 0,372 & 0,147 \\
\hline Día 2 C & 8,387 & 0,099 & 8,339 & 0,083 & 0,048 \\
\hline Día 3 C & 8,403 & 0,040 & 8,338 & 0,060 & 0,066 \\
\hline Día 4 C & 8,347 & 0,046 & 8,420 & 0,095 & $-0,073$ \\
\hline Día 5 C & 8,333 & 0,021 & 8,309 & 0,138 & 0,025 \\
\hline Día 1 । & 8,459 & 0,076 & 8,377 & 0,091 & 0,081 \\
\hline Día 2 I & 8,304 & 0,141 & 8,423 & 0,155 & $-0,119$ \\
\hline Día 3 l & 8,265 & 0,097 & 8,383 & 0,114 & $-0,118$ \\
\hline Día 4 I & 8,253 & 0,084 & 8,291 & 0,219 & $-0,037$ \\
\hline Día 5 । & 8,200 & 0,123 & 8,317 & 0,105 & $-0,117$ \\
\hline Día 6I & 7,363 & 1,475 & 7,645 & 1,145 & $-0,282$ \\
\hline Día 7I & 6,150 & 0,000 & 7,041 & 0,000 & $-0,891$ \\
\hline
\end{tabular}

Figura 3. Barras del comportamiento del pH urinario durante las fases de control e inducción de la alcalosis metabólica hipoclorémica, en las que se puede apreciar la disminución marcada en el pH en las últimas 48 h de la fase de inducción, lo cual muestra el desarrollo del fenómeno de aciduria paradójica

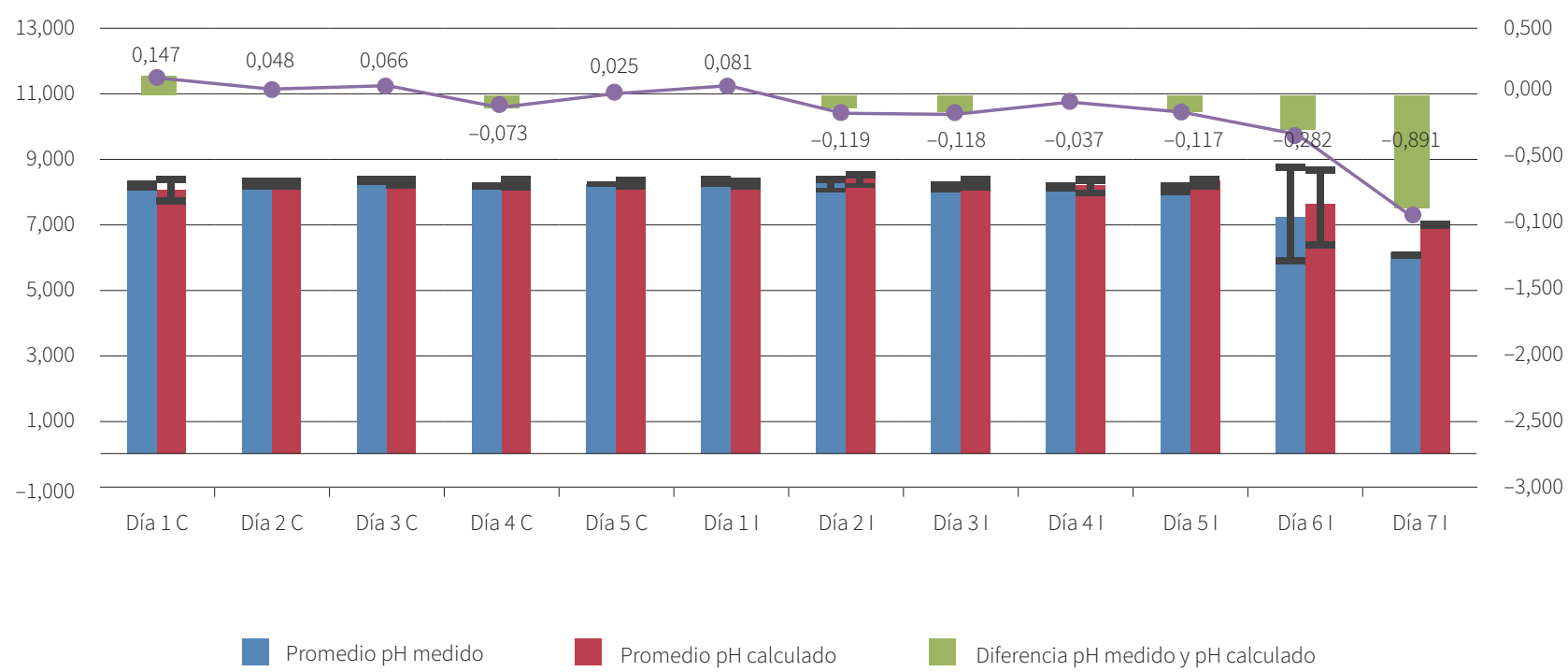


Los resultados de la prueba de Friedman $(\mathrm{p} \leq 0,05)$ sobre diferencias significativas entre el $\mathrm{pH}$ urinario calculado y medido durante las fases de control e inducción de la alcalosis metabólica indican que durante el periodo de control $(0,201)$ no existe diferencia entre las dos variables, pero durante la inducción $(0,0)$ sí se evidencian diferencias. A pesar de estas diferencias, la correla- ción de Pearson mostró un valor de $\mathrm{r}=0,874(\mathrm{p} \leq 0,01)$, lo cual indica una alta correlación entre el $\mathrm{pH}$ calculado y el pH medido, lo que se puede apreciar en el gráfico de dispersión de la figura 4, que confirma la aplicación de la teoría de iones fuertes en ovinos con alcalosis metabólica hipoclorémica.

Figura 4. Dispersión de datos según el coeficiente de correlación de Pearson, que muestra la concentración de datos y confirma la asociación entre el pH medido y el calculado con la aplicación de la teoría de iones fuertes en la orina

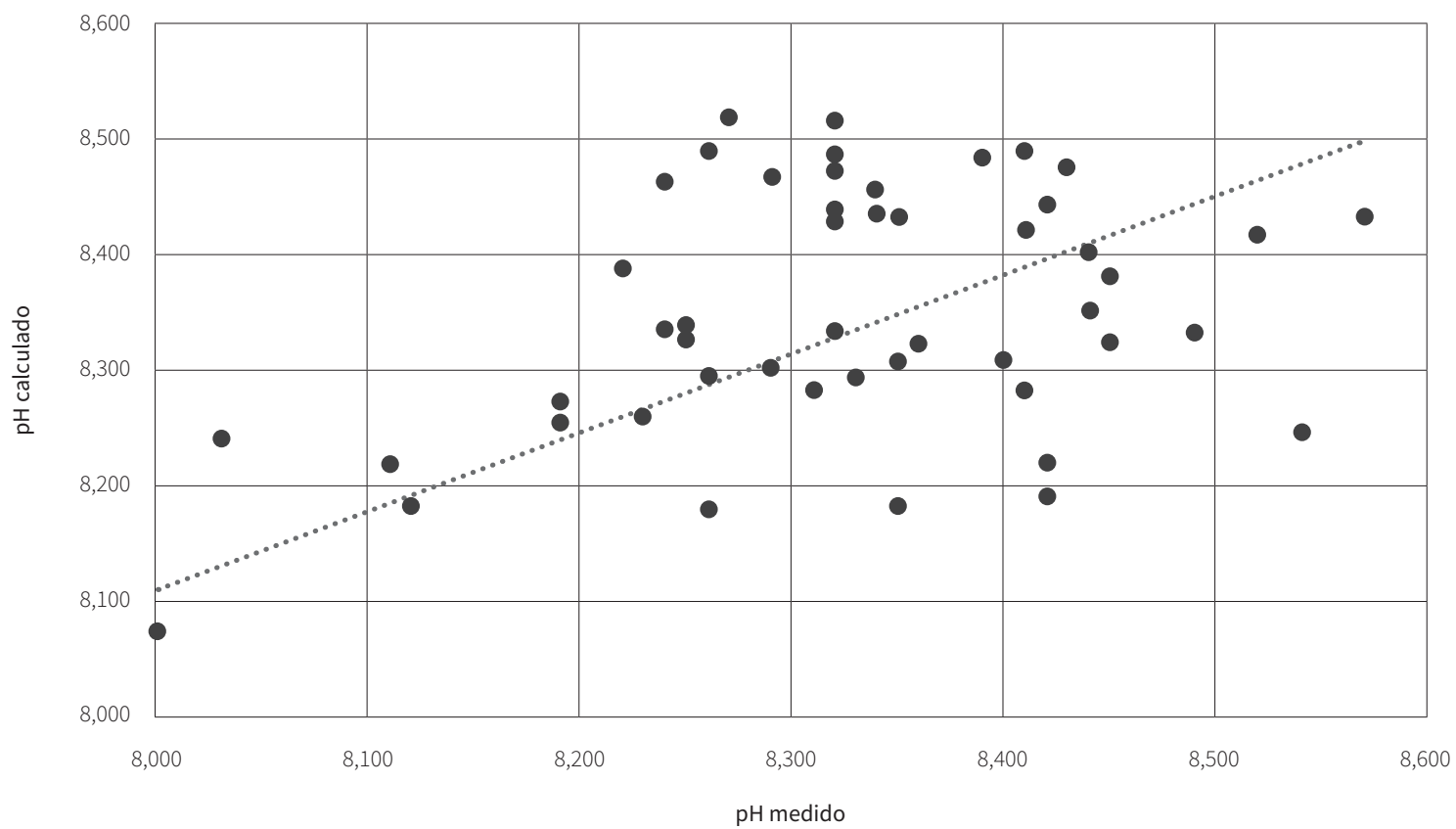

Cuando se calculó el SID urinario con la ENB, se encontró que mientras se mantuvo un $\mathrm{pH}$ urinario alcalino, este mostró valores entre 208,779 (DE 54,943) y 133,671 (DE 63,568) mmol/L. Sin embargo, el valor bajó drásticamente cuando se presentó el fenómeno de aciduria, con promedios entre $-7,900(\mathrm{DE} 1,061)$ y 98,433 (DE 91,73) mmol/L.Este comportamiento también mostró la ENB, pero en sentido inverso, mientras se mantuvo el $\mathrm{pH}$ alcalino. De esta manera, el promedio mínimo observado fue de -131,071 (DE 69,481) $-206,179$ (DE 59,700) $\mathrm{mmol} / \mathrm{L}$.
El valor de la correlación de Pearson entre el SID calculado y el $\mathrm{pH}$ calculado fue de $\rho=0,838$, mientras la correlación de Pearson entre el SID y el pH medido presentó un valor de $\rho=0,588$. Esto muestra una fuerte correlación entre el SID calculado y el $\mathrm{pH}$ calculado, pero una media correlación entre el SID calculado y el pH medido. Los valores mencionados se pueden apreciar en el gráfico de dispersión, en el caso del SID y el $\mathrm{pH}$ calculado. En la figura 5 se observa una clara tendencia, pero esta no se aprecia de la misma manera en el SID y el pH medido en la figura 6 . 
Figura 5. Dispersión de datos según el coeficiente de correlación de Pearson que muestra la concentración de datos y confirma la asociación entre el SID calculado con la ENA y el pH calculado, lo cual evidencia la asociación entre estas variables y la importancia del SID como determinante del pH urinario en ovinos con alcalosis metabólica hipoclorémica

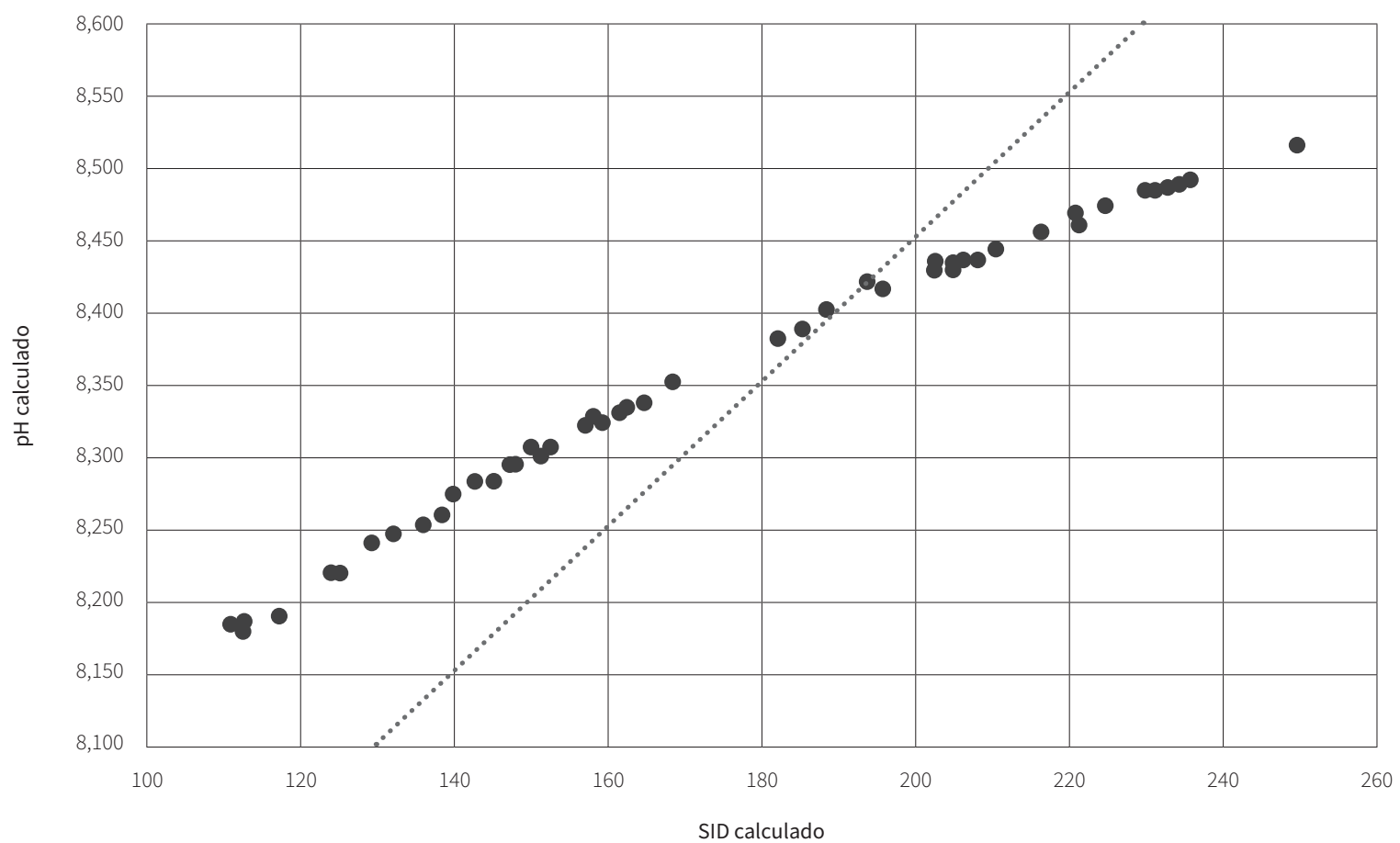

Figura 6. Dispersión de datos según el coeficiente de correlación de Pearson, que muestra la concentración de datos y confirma la asociación entre el SID calculado con la ENA y el pH medido, lo cual evidencia la asociación entre estas variables y la importancia del SID como determinante del pH urinario en ovinos con alcalosis metabólica hipoclorémica

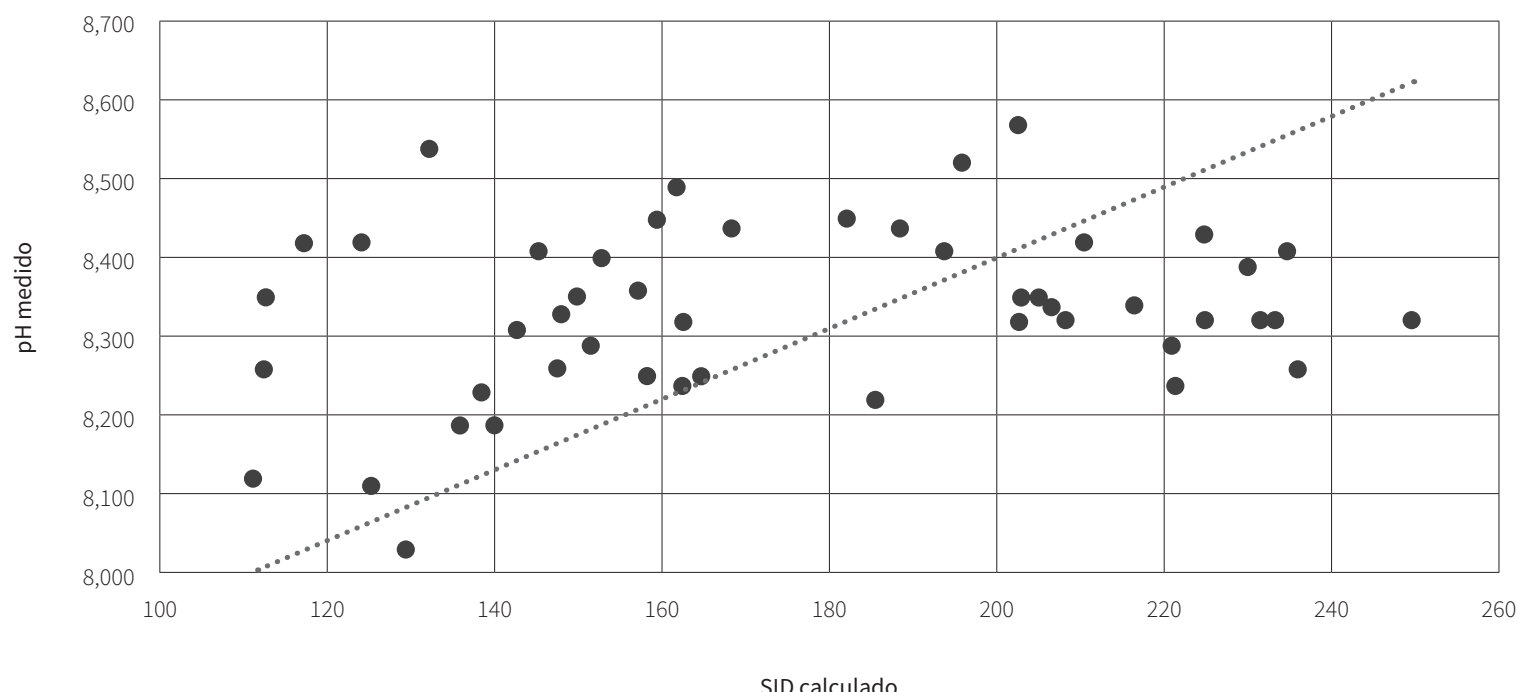


En el caso del amonio, se evidenció un claro aumento en las concentraciones de este metabolito cuando la orina mostró el fenómeno de aciduria. Se encontraron concentraciones promedio entre 4,667 (DE 3,166) y 16,250 (DE 0) $\mathrm{mmol} / \mathrm{L}$. Dicho comportamiento de aumento en las concentraciones mientras se desarrolla la aciduria es evidente también con la acidez titulable $-100,500$ (DE 88,556) - -5,750 (DE 0) $\mathrm{mmol} / \mathrm{L}$.
En la tabla 2 se muestran los promedios de la acidez titulable, la concentración de amonio, la ENB y la diferencia de iones fuertes para la orina de los sujetos experimentales durante las fases de control y de inducción de la alcalosis metabólica. Cabe resaltar que aquellos sujetos experimentales que desarrollaron aciduria durante el experimento muestran una disminución marcada en el SID, como se muestra en la figura 7 , y aumento en los valores de amonio, acidez titulable y ENB.

Tabla 2. Promedios de AT, $\mathrm{NH}_{4}$, ENA y diferencia de iones fuertes (SID) urinario durante la fase experimental de control correspondiente a 5 días y la fase de inducción de la alcalosis metabólica hipoclorémica correspondiente a 7 días

\begin{tabular}{|c|c|c|c|c|c|c|c|c|}
\hline Fase & AT & $\sigma$ & $\mathrm{NH}_{4}$ & $\sigma$ & ENA & $\Sigma$ & SID & $\sigma$ \\
\hline Día 1 C & $-133,250$ & 62,430 & 2,179 & 1,344 & $-131,071$ & 69,481 & 133,671 & 63,568 \\
\hline Día 2 C & $-165,750$ & 35,058 & 2,625 & 1,506 & $-163,125$ & 36,029 & 165,725 & 36,029 \\
\hline Día 3C & $-163,583$ & 23,474 & 2,667 & 1,283 & $-160,917$ & 22,328 & 163,517 & 22,328 \\
\hline Día 4 C & $-200,000$ & 41,354 & 1,750 & 0,433 & $-198,250$ & 41,569 & 200,850 & 41,569 \\
\hline Día 5 C & $-157,250$ & 52,007 & 2,667 & 1,443 & $-154,583$ & 53,390 & 157,183 & 53,390 \\
\hline Día 11 & $-181,536$ & 33,660 & 1,893 & 0,967 & $-179,643$ & 35,817 & 182,243 & 33,242 \\
\hline Día 21 & $-207,679$ & 54,308 & 1,500 & 1,155 & $-206,179$ & 59,700 & 208,779 & 54,943 \\
\hline Día 31 & $-185,750$ & 45,512 & 2,583 & 2,990 & $-183,167$ & 46,870 & 185,767 & 46,870 \\
\hline Día 4 I & $-159,667$ & 65,551 & 10,542 & 21,835 & $-149,125$ & 83,162 & 151,725 & 83,162 \\
\hline Día 51 & $-158,688$ & 42,447 & 1,938 & 1,106 & $-156,750$ & 42,026 & 159,350 & 42,026 \\
\hline Día 61 & $-100,500$ & 88,556 & 4,667 & 3,166 & $-95,833$ & 91,713 & 98,433 & 91,713 \\
\hline Día 7 I & $-5,750$ & 0,000 & 16,250 & 0,000 & 10,500 & 0,000 & $-7,900$ & 1,061 \\
\hline
\end{tabular}


Figura 7. Comportamiento del SID durante las fases de control e inducción de la alcalosis metabólica hipoclorémica, en el que se puede apreciar el descenso marcado en los valores de esta variable determinante del pH al momento de la generación de la aciduria paradójica y que explica el porqué del descenso en el pH urinario

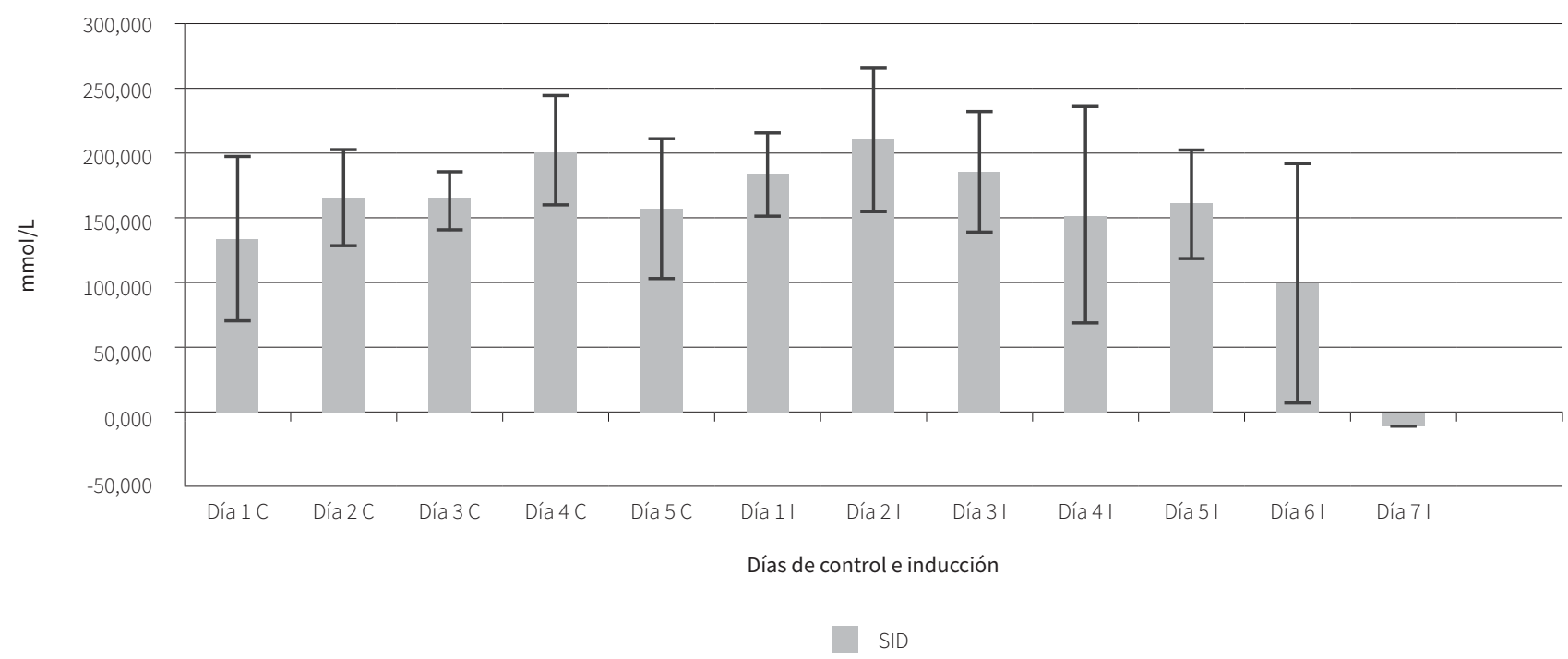

\section{Discusión}

El hecho de que se haya extendido la fase de control en tres de los sujetos experimentales se pudo deber al tipo de cánula que se implementó en el desarrollo del estudio, ya que las cánulas en forma de $\mathrm{T}$, como la usada en el presente modelo experimental, se encuentran diseñadas para evaluación de componentes nutricionales en la dieta de rumiantes $(19,20)$. Los trabajos realizados en el análisis metabólico de estado ácido base usan modelos con cánulas de reentrada, y obstruyen el paso del contenido al intestino delgado. Estos modelos garantizan una completa pérdida de contenido o bloqueo en el paso de este, al momento deseado; mientras que con las cánulas en forma de $\mathrm{T}$ se presenta taponamiento por el contenido alimenticio, debido al diámetro de estas, evento que dificulta el adecuado manejo de la condición deseada. Sin embargo, la liberación de contenido abomasal es abundante mientras se garantice que exista un flujo adecuado y continuo (20,26-28).
Por otra parte, las cánulas en forma de $\mathrm{T}$ permiten el paso de contenido alimenticio por sus bordes externos, hecho que posiblemente extiende los tiempos en el desarrollo de la alcalosis metabólica hipoclorémica, y desde luego hacen más lenta la compensación renal. Esto explica el hecho de que en el actual modelo experimental el desarrollo de la aciduria se tomara entre 6 y 7 días, mientras en otros modelos tan solo se demora entre 2,5 a 4 días. Es posiblemente por este mismo evento que no todos los sujetos experimentales desarrollaron dicha alteración antes de su deterioro físico y la realización de la eutanasia $(24,26,27)$.

A pesar de lo mencionado, se decide realizar el presente estudio con las cánulas en forma de $\mathrm{T}$, ya que en el país no se cuenta con cánulas de reentrada y no fue posible su consecución para el estudio por motivos de presupuesto; además, no sería posible la obstrucción completa del contenido intestinal por conflictos éticos. 
El comportamiento del $\mathrm{pH}$ en la orina se mantuvo alcalino durante casi toda la fase experimental, como se encuentra registrado en diferentes estudios sobre la alcalosis metabólica hipoclorémica, y solo descendió a concentraciones ácidas en la últimas $48 \mathrm{~h}$ del experimento, seguramente como consecuencia del desorden electrolítico y la compensación frente a dichos estados. Sin embargo, en el presente estudio, aunque se midieron los electrolitos, y se tuvieron en cuenta los resultados obtenidos con los equipos con que se contaba, las mediciones no fueron confiables, y los resultados fueron descartados; por lo tanto, no se pudieron medir las alteraciones electrolíticas que explicarían acertadamente el fenómeno que se presenta cuando el $\mathrm{pH}$ desciende. Por tal razón, solo se podría especular sobre las causas de dicho descenso $(26,28,29)$. No obstante, se determinó el SID de manera indirecta, utilizando la fórmula sugerida por Constable y colaboradores en 2009 (9), que incluye la ENA y que a su vez representa la diferencia electrolítica.

A pesar de observarse una diferencia aparentemente amplia entre los valores absolutos medidos y calculados del $\mathrm{pH}$, la prueba de Friedman confirmó que durante el control no existieron diferencias significativas $(\mathrm{p} \leq 0,05)$ entre el $\mathrm{pH}$ medido y el calculado. Sin embargo, esta diferencia fue apreciable durante el periodo de inducción de la alcalosis metabólica, muy seguramente debido a los desórdenes electrolíticos que se presentan durante este periodo experimental. Pero el análisis de correlación de Pearson $(\rho=0,874[p \leq 0,01])$ evidencia una alta correlación entre los valores calculados y los medidos. Ello sugiere que a pesar de encontrarse una aparente diferencia entre estos valores, sobre todo durante la fase final de inducción de la alcalosis metabólica, dicha diferencia no altera la predicción del $\mathrm{pH}$, y seguramente se da como consecuencia del desorden electrolítico que se está presentando en el plasma y su efecto en la zona renal (9).

Por otra parte, la correlación de Pearson confirma los hallazgos de Constable y colaboradores (9), quienes sustentan que el $\mathrm{pH}$ urinario de los bovinos es determinado por el SID urinario, la concentración de amonio y la $\mathrm{PCO}_{2}$, lo que obtuvieron aplicando la ecuación descrita por para $\mathrm{pH}$ urinario entre 5 y 8 . En el presente estudio dicha ecuación fue igualmente valedera para ovinos que manejan el pH urinario dentro de estos rangos, y se aplican las mismas variables independientes. Por lo tanto, en el caso de ovinos se confirma, de esta manera, que la concentración de bicarbonato no afecta directamente el $\mathrm{pH}$, y por ello es considerada una variable dependiente de las ya descritas variables independientes (9).

El hecho de que exista una correlación mayor entre el SID y el $\mathrm{pH}$ calculado que para el medido puede deberse a que el $\mathrm{pH}$ calculado es tomado a partir de diferentes variables que desde el punto de vista teórico son las generadoras del valor real. Sin embargo, siempre existirá cierto grado de error al momento de los cálculos, lo cual no permite obtener un valor idéntico sino más bien muy cercano al esperado, que es lo que finalmente se encontró con la alta correlación entre el $\mathrm{pH}$ calculado y medido. Además de esto, solo se está correlacionando una variable, y, como ya se sabe, según la teoría de diferencia de iones fuertes en la orina, son el SID y los ácidos débiles no volátiles los que mayormente afectan este valor. Por lo tanto, esta correlación muestra que otros factores contribuyen al mantenimiento del $\mathrm{pH}$ en los valores medidos, siendo el SID uno de los más importantes (9).

En este estudio no fue posible la determinación directa del SID, debido a la no confiabilidad de las mediciones de los electrolitos. Sin embargo, el SID se obtuvo utilizando la fórmula matemática descrita por Constable y colaboradores en 2009 (9), en la cual el SID es igual a $(\mathrm{SID}=\mathrm{ENB}+2,6=-\mathrm{ENA}+2,6)$, donde la ENA se obtuvo por la titulación de la orina. Los valores del $\mathrm{pH}$ calculado se obtuvieron usando los valores de este SID. Los resultados muestran un grado importante de confiabilidad gracias a la alta correlación entre el $\mathrm{pH}$ urinario medido y el $\mathrm{pH}$ urinario calculado $(\mathrm{p} \leq 0,01)$. Esta fórmula es aplicable para rangos de $\mathrm{pH}$ entre 5 y 8 , lo cual permite determinar el $\mathrm{pH}$ urinario cuando no se cuenta con los equipos necesarios para la medición de electrolitos, y se aplica la teoría de iones fuertes en la orina de ovinos (9). Esto, desde el punto de vista tanto investigativo como clínico, facilita la interpretación del estado ácido base sin necesidad de realizar la medición de cada uno de los electrolitos implicados. 
Cuando se aplicó la anterior fórmula matemática para el cálculo del valor del SID, se encontró que en el momento en que se presentaba el fenómeno de aciduria en la orina de pacientes con alcalosis metabólica hipoclorémica descendían drásticamente los valores de SID; según la teoría de iones fuertes o el análisis físico-químico, en condiciones alcalinas los valores de este parámetro son altos, mientras que en condiciones ácidas estos valores descienden. Por lo tanto, el comportamiento del SID durante el experimento es concordante con lo estipulado en esta teoría. Adicionalmente, no se cuenta con parámetros de referencia de estos valores de SID, con los cuales comparar los actuales resultados, pero su comportamiento, como ya se comprobó, corresponde al esperado $(5,6,9,22,30,31)$. La aplicación de esta fórmula permitirá realizar de manera económica y práctica el cálculo del SID, lo cual es importante en diferentes investigaciones que implican el uso de este modelo y la medición de esta variable, como en el cálculo de las dietas catiónicas para la prevención de enfermedades metabólicas en rumiantes.

Existen diferentes estudios sobre el efecto directo del escape del $\mathrm{CO}_{2}$ en la medición del $\mathrm{pH}$. Algunos autores sugieren que este puede afectar incrementando 0,5 unidades sobre el $\mathrm{pH}$ real. Sin embargo, otros estudios exponen que este solo incrementa el $\mathrm{pH}$ urinario en 0,06 unidades. En el presente estudio se decidió asumir que la $\mathrm{PCO}_{2}$ era constante, y por lo tanto no afectaba ni la medición ni el cálculo del pH (9); y como se evidenció, existe una alta correlación entre el $\mathrm{pH}$ calculado y el medido, lo que permite sustentar que la asunción de una presión de $\mathrm{CO}_{2}$ constante es valedera para la realización de los cálculos del pH, y que el $\mathrm{CO}_{2}$ no afecta de manera significativa dichos cálculos. Lo anterior, por ende, permite inferir que posiblemente los grandes involucrados en la determinación del $\mathrm{pH}$ son el SID y la concentración de amonio (9).

Cuando el pH urinario se encuentra alcalino, la excreción de amonio es casi cercana a 0 , como se evidencia en diferentes estudios $(25,32)$. Esto explica por qué los valores encontrados en la concentración de amonio antes de la presentación del fenómeno de aciduria fueron bajos. En el presente estudio, los valores de amonio se incrementaron significativamente durante la aciduria en los sujetos experimentales; este aumento ha sido relacionado con la disminución en la concentración de $\mathrm{K}^{+}$, disminución que ocurre posiblemente por un estímulo de la reabsorción de $\mathrm{K}^{+}$, lo cual generaría, desde el punto de vista físico-químico, una disminución en el valor del SID, y de esta manera una disminución del $\mathrm{pH}$ urinario, lo que estimularía la producción de amonio en túbulos renales. Esta hipótesis no se pudo comprobar, ya que no fue posible la medición de electrolitos y el cálculo directo del SID. Sin embargo, esta inferencia es la presunción más cercana a la explicación de este comportamiento en las concentraciones de amonio (9,26,32-34).

Cabe resaltar, en este punto, que Constable y colaboradores (9) proponen que las concentraciones de $\mathrm{K}^{+}$tienen un efecto marcado en el $\mathrm{pH}$ urinario en bovinos. Proponen, además, que cuando existe una excreción alta de $\mathrm{K}^{+}$en la orina, el $\mathrm{pH}$ se torna alcalino, y que cuando estas bajan, el pH urinario es ácido, basado en la ecuación para predecir el $\mathrm{pH}$ en rangos entre 5 y 8 . Teniendo en cuenta que la concentración de $\mathrm{K}^{+}$es dependiente del consumo y del estado ácido base, en el presente estudio, en el cual los sujetos experimentaron la alcalosis metabólica y desarrollaron anorexia, se podría explicar la presencia de aciduria por las concentraciones bajas de $\mathrm{K}^{+}$en la orina. Sin embargo, dicha hipótesis no pudo ser comprobada debido a la no determinación de las concentraciones electrolíticas de $\mathrm{K}^{+}$en la orina $(9,33,34)$.

Finalmente, se puede concluir del presente estudio que el $\mathrm{pH}$ calculado urinario, según el aboramiento físicoquímico, es muy similar al pH medido en la orina de ovinos con alcalosis metabólica hipoclorémica. Por otra parte, también se puede señalar la importancia del SID como determinante del $\mathrm{pH}$, dada la alta correlación entre esta variable y el valor del $\mathrm{pH}$. 


\section{REFERENCIAS}

1. Anderson R, Pickering E. Some aspects of the renal excretion of bicarbonate in the cow. Procced Physiol. 1962;31-2.

2. Belge A, Bakir B, Özçelik A. A technique of duodenal cannulation in sheep. Small Rumin Res. 2002;44(2):167-71. doi: https://doi.org/10.1016/ S0921-4488(02)00052-4

3. Chan JCM. The rapid determination of urinary tritratable acid and ammonium and evaluation of freezing as a method of preservation. Clin Biochem. 1972;5(1-4):94-8. doi: https://doi.org/10.1016/S00099120(72)80014-6

4. James CM, Chan MD. Urinary organic anions: clinical significance and evaluation of a method for determination and preservation. Clin Biochem. 1972;5(1-4):182-5. doi: https://doi.org/10.1016/ S0009-9120(72)80029-8

5. Constable PD. A simplified strong ion model for acidbase equilibria: application to horse plasma. J Appl Physiol (1985). 1997;83(1):297-311.

6. Constable PD. Clinical assessment of acid-base status. Strong ion difference theory. Vet Clin North Am Food Anim Pract. 1999;15(2):447-71. doi: https://doi. org/10.1016/S0749-0720(15)30158-4

7. Constable PD. Clinical assesment of acid-base status: comparison of the Henderson-Hasselbalch and strong ion approaches. Vet Clin Path. 2000;29(4):115-28. doi: https://doi.org/10.1111/j.1939-165X.2000.tb00241.x

8. Constable P. Fluid an electrolyte therapy in ruminants. Vet Clin North Am Food Anim. 2003;19(3):557-97. doi: https://doi.org/10.1016/S0749-0720(03)00054-9

9. Constable P, Gelfert C, Furll M, Staufenbel R, Staempfli $\mathrm{H}$. Application of strong ion difference theory to urine and relationship between urine $\mathrm{pH}$ and net acid excretion in cattle. Am J Vet Res. 2009;70(7):915-25. doi: https://doi.org/10.2460/ajvr.70.7.915

10. DiBartola $S$. Terapéutica de líquidos en pequeñas especies. México: McGraw-Hill; 2002.

11. Edwards SL. Pathophysiology of acid base balance. The theory practice relationship. Intensive Crit Care Nurs. 2008;24(1):28-38. doi: https://doi.org/10.1016/j. iccn.2007.05.003
12. Fencl V, Rossing T. Acid-base disorders in critical care medicine. Annu Rev Med. 1989;40:17-29. doi: https:// doi.org/10.1146/annurev.me.40.020189.000313

13. Fencl V, Leith D. Stewart's quantitative acid base chemistry: application in biology and medicine. Respir Physiol. 1993;91(1):1-16. doi: https://doi. org/10.1016/0034-5687(93)90085-O

14. Foy D, Morais H. Metabolic alkalosis: A quick reference. Vet Clin North Am Small Anim Pract. 2008;38(3):435-8. doi: https://doi.org/10.1016/j. cvsm.2008.01.023

15. Fubini S, Smith D, Grohn Y, Levine S, Devel D. Replacement of chloride deficit by use of $1.8 \% \mathrm{NaCl}$ to correct experimentally induced hypochloremic metabolic alkalosis in sheep. Am J Vet Res. 1991;52(11):1898-902.

16. Galla J. Metabolic alkalosis. J Am Soc Nephrol. 2000;11(2):369-75.

17. Gingerich D, Murdick P. Paradoxic aciduria in bovine metabolic alkalosis. J Am Vet Med Assoc. 1975;166(3):227-30.

18. Guyton A, Hall J. Textbook of medical physiology. Philadelphia: Elsevier; 2011.

19. Harmon DL, Richards CJ. Considerations for gastrointestinal cannulations in ruminants. J Anim Sci. 1997;75(8):2248-55. doi: https://doi. org $/ 10.2527 / 1997.7582248 x$

20. Ivan M, Johnston D. Reentrant cannulation of the small intestine in sheep: cannula and surgical method. J Anim Sci. 1981;52(4):849-56. doi: https://doi. org/10.2527/jas1981.524849x

21. Jörgensen K. Titrimetric determination of the net excretion of acid/base in urine. Sacandinav J Clin Lab Investigation. 1957;9(3):287-91. doi: https://doi. org/10.3109/00365515709079972

22. Kellum J, Elbers P. Stewart's textbook of acid base. Amsterdam: Lulu.com; 2009.

23. Komarek RJ. Intestinal cannulation of cattle and sheep with a T-shaped cannula designed for total digesta collection without externalizing digesta flow. J Anim Sci. 1981;53(3):796-802. doi: https://doi.org/10.2527/ jas1981.533796x

24. Smith DF, Lunn DP, Robinson GM, McGuirk SM, Nordheim EV, MacWilliams PS. Experimental model of hyochloremic metabolic alkalosis caused by diver- 
sion of abomasal outflow in sheep. Am J Vet Res. 1990;51(11):1715-22.

25. Van Slike DD, Palmer WW. Studies of acidosis XVI: The tritration of organic acids in urine. J Biol Chem. 1920;41:567-85.

26. Lunn DP, McGuirk SM, Smith DF, MacWilliams PS. Renal net acid and electrolyte excretion in an experimental modelo of hypochloremic metabolic alkalosis in sheep. Am J Vet Res. 1990;51(11):1723-31.

27. Papadopoulos P, Raptopoulos D, Dessiris A, Tsimopoulos G. Experimental intestinal obstruction in cattle part I: changes in the clinical picture. Zentralbl Veterinarmed A. 1985;32(4):264-75. doi: https://doi. org/10.1111/j.1439-0442.1985.tb01943.x

28. Papadopoulos P, Raptopoulos D, Dessiris A, Tsinopoulos G. Experimental intestinal obstruction in cattle part II: changes in blood. Urine and rumen content chemistry. Zentralbl Veterinarmed A. 1985;32(4):276-88. doi: https://doi.org/10.1111/j.1439-0442.1985.tb01944.x

29. McGuirk S, Butler D. Metabolic alkalosis with paradoxic aciduria in cattle. J Am Vet Med Assoc. $1980 ; 177(6): 551-4$.
30. Stewart PA. Modern quantitative acid-base chemistry. Can J Physiol Pharmacol. 1983;61(12):1444-61. doi: https://doi.org/10.1139/y83-207

31. Stewart P. How to understand acid-base. A quantitative acid-base primer for biology and medicine. Nueva York: Elsevier; 1981.

32. Lunn DP, McGuirk SM. Renal regulation of electrolyte and acid-base balance in ruminants. Vet Clin North Am Fodd Anim Pract. 1990;6(1):1-28. doi: https://doi. org/10.1016/S0749-0720(15)30891-4

33. Scott D. The effects of intravenous infusion of $\mathrm{kcl}$ or hcl on the renal excretion of potassium in sheep. QJ Exp Physiol Cogn Med Sci. 1969;54:25-35. doi: https://doi. org/10.1113/expphysiol.1969.sp002001

34. Scott D. Renal excretion of potassium and acid by sheep. QJ Exp Physiol Cogn Med Sci. 1969;54:41222. doi: https://doi.org/10.1113/expphysiol.1969. sp002040 